\title{
Interpretation of Tc-99m sestamibi parathyroid SPECT-CT scans made easy for better surgical outcomes in patients with primary hyperparathyroidism
}

This article was published in the following Dove Press journal:

Reports in Medical Imaging

23 December 2016

Number of times this article has been viewed

\author{
Isis W Gayed' \\ Ron J Karni² \\ David Q Wan' \\ Jeena $M$ Varghese ${ }^{3}$ \\ Kelly L Wirfel ${ }^{3}$ \\ Kyoung S Won ${ }^{4}$ \\ Usha A Joseph' \\ 'Nuclear Medicine Section, \\ Department of Diagnostic and \\ Interventional Imaging, ${ }^{2}$ Department \\ of Otorhinolaryngology, Head and \\ Neck Surgery, ${ }^{3}$ Department of \\ Internal Medicine - Endocrinology, \\ Diabetes and Metabolism, Medical \\ School at Houston Health Science \\ Center, Houston, TX, USA; \\ ${ }^{4}$ Department of Nuclear Medicine, \\ Keimyung University Dongsan Medical \\ Center, Daegu, South Korea
}

Correspondence: Isis W Gayed Department of Radiology, University of Texas-Houston, Medical School, 643I

Fannin, Suite 2.130b, Houston, TX 77030, USA

Tel+I 7I3704 I789

Fax+I 7I3 7041596

Email Isis.W.Gayed @uth.tmc.edu
Objective: This study aims to evaluate the accuracy of Tc-99m sestamibi parathyroid scan (SPS) with single-photon emission computerized tomography/computerized tomography (SPECT/CT) in patients with primary hyperparathyroidism after optimizing the interpretation of the scans with a systematic and simple method for localization of the abnormal parathyroid gland.

Materials and methods: Consecutive patients, who underwent SPS followed by surgical intervention, were included. Data were collected retrospectively including patients' demographics, serum calcium and parathyroid hormone (PTH) levels, SPS findings, and surgical and pathological findings. Our optimized systematic method of interpretation of SPS starts by reviewing initial and 3 hours delayed planar images; subsequently we reviewed coronal and axial SPECT/CT images. A simple clockwise localization of the abnormal gland in relation to the trachea and its level in relation to the thyroid in a cranio-caudal level facilitates intraoperative localization of parathyroid adenomas.

Results: A total of 53 patients were included; 9 males and 44 females with an average age of $58.3 \pm 14$ years. The average preoperative serum calcium and PTH were $10.5 \mathrm{mg} / \mathrm{dL}$ and 165.4 $\mathrm{pg} / \mathrm{m}$, respectively. The median interval between the scan and operative resection was 50 days. Our SPS interpretation method correlated perfectly with the surgical finding in 48/53 patients (90.6\%); it was on the correct side of the surgical finding but not the exact location in $3 / 53$ (5.7\%) and nonlocalizing in 2/53 (3.7\%). The average intraoperative PTH after resection of the adenoma was $39.7 \mathrm{pg} / \mathrm{mL}$.

Conclusion: Our optimized method for interpretation of SPS demonstrated high accuracy with good surgical outcomes.

Keywords: parathyroid scan, Tc-99 sestamibi, primary hyperparathyroidism, SPECT-CT, parathyroid adenoma

\section{Introduction}

Primary hyperparathyroidism (PHPT) is the third most common endocrine disorder after diabetes and hyperthyroidism. ${ }^{1}$ Involvement of one parathyroid gland is the most common presentation of PHPT occurring in $80 \%-85 \%$ of patients. ${ }^{1,2}$ Most patients are either asymptomatic or have vague nonspecific symptoms. The disease is usually found on routine serum calcium measurements. However, approximately one-third of the patients 
usually become symptomatic over a 15 -year period. ${ }^{3}$ Operative resection of the parathyroid adenoma is the only curative treatment in PHPT. Parathyroidectomy has evolved significantly over the past 3 decades. A major 4 glands neck exploration and intraoperative palpation in attempt to identify and resect the parathyroid adenoma have changed to minimally invasive procedure with significant decrease in the size of the neck scar and postoperative morbidity. ${ }^{4-6}$ This major evolution of parathyroidectomy is the result of significant advancement in parathyroid imaging. Multiple imaging modalities are presently available to help preoperative precise localization of parathyroid adenomas including radionuclide imaging, ultrasound, ${ }^{7-9}$ four dimensional computerized tomography (4D CT),${ }^{10}$ and/or magnetic resonance imaging (MRI). ${ }^{11}$ Intraoperative localization can also be enhanced with intraoperative ultrasound, intraoperative injection of Tc-99m sestamibi for gamma probe localization, or intraoperative MRI. ${ }^{12-14}$ Resection of the pathologic parathyroid adenoma is confirmed with intraoperative reduction of parathyroid hormone (PTH) level of $\geq 50 \%$ of the baseline level preoperatively. ${ }^{15,16}$

Radionuclide imaging has also improved significantly over the past 2 decades. It started with the dual-tracers sequential subtraction parathyroid scintigraphy which was difficult to perform requiring patient compliance with no motion throughout the study. ${ }^{17}$ This was followed by the more robust single tracer dual-phase protocols using Tc-99m sestamibi ${ }^{17,18}$ or Tc-99m tetrofosmine ${ }^{19}$ with better accuracy of parathyroid adenoma or hyperplasia detection. The addition of single-photon emission computerized tomography (SPECT) and subsequently SPECT/CT has improved the detection rate and localization of the abnormal parathyroid gland significantly. ${ }^{20-23}$ Simultaneous dual tracer imaging with a thyroid specific tracer like I-123 with the Tc-99m sestamibi is thought to decrease imaging time and decrease false positive studies in patients with coexisting thyroid abnormalities. ${ }^{23}$

Most institutions will perform at least 2 imaging modalities to confirm the presence and location of an abnormal parathyroid gland. This is usually a combination of ultrasound and parathyroid scan. ${ }^{24,25}$ Additional imaging modalities as MRI or 4D CT may be thought for discordant or inconclusive results between US and parathyroid scans. ${ }^{10}$

Our study evaluated the accuracy and outcomes from a standardized simple method of interpretation and reporting of Tc-99m sestamibi dual-phase scan with SPECT/CT as the imaging modality for the diagnosis and localization of parathyroid adenoma in patients with PHPT. After a long experience with interpretation of SPECT and SPECT/CT as part of parathyroid scans, we have developed a systematic approach to the interpretation of parathyroid scans, which we believe can contribute to higher accuracy in identifying the abnormal parathyroid gland. Additionally, our method of reporting the location of the parathyroid adenoma facilitates clear and simple standard mean of communication with the surgeons of the location of the abnormal parathyroid gland with favorable surgical outcomes. Our method of interpretation and reporting of Tc-99m sestamibi dual-phase scan with SPECT/CT can help other imagers and labs to improve on their accuracy for diagnosis and localization of parathyroid adenomas.

\section{Materials and methods}

After obtaining the UT Health-Memorial Hermann Hospital Institutional Review Board approval to conduct this study. Since our study is a retrospective study obtaining a consent form from the patients was waived by the IRB. We retrospectively included all consecutive patients who underwent Tc-99m sestamibi parathyroid scans (SPS) with SPECT/CT followed by parathyroidectomy between April 2012 and April 2014. Data collected included patients' demographics, serum calcium levels, preoperative and intraoperative PTH levels, parathyroid scan findings from reports, interval between parathyroid scan and parathyroidectomy, and surgical and pathological findings.

\section{Systematic interpretations of parathyroid scans}

After a long experience and open communication with our experienced surgeons performing the parathyroidectomies, our two experienced nuclear medicine physicians developed a systematic approach to the interpretation of parathyroid scans with SPECT/CT. The steps for our systematic interpretations are as follows:

- We conduct an initial review of 20 minutes and 2-3 hours delayed planer images of the neck and chest obtained in the anterior and bilateral anterior oblique positions. Any foci of increased tracer uptake regardless of their intensity are noted for further evaluation on the SPECT/CT images. Faint foci of uptake may occasionally be seen on oblique images of the neck rather than on the anterior planar image. Focal increased uptake seen on early images may or may not show retention on delayed planer images in parathyroid adenomas but will direct the readers' attention to the side of possible adenoma. We also look for possible ectopic parathyroid adenomas between the thyroid gland and floor of the mouth or submandibular glands as well as between the heart and the thyroid gland.

- Subsequently, we review the coronal SPECT/CT slices to confirm the presence of focal uptake and its laterality and 
relative location to the thyroid lobes in a cranio-caudal level. It is better to start from anterior slices of the thyroid while observing for any focal uptake below the thyroid lobes or asymmetric elongation of one thyroid lobe inferiorly which are the typical appearances of an inferior parathyroid adenomas. Continuous slow scrolling through the thyroid gland will help to differentiate thyroid versus parathyroid adenomas. A superior parathyroid adenoma will usually appear as focal tracer concentration in the immediate few slices posterior to the thyroid gland at different cranio-caudal levels compared with the thyroid gland since most of the superior parathyroid glands tend to grow inferiorly in the tracheoesophageal groove.

- Finally, the axial SPECT/CT slices are reviewed for more precise localization of the abnormal parathyroid gland in relation to surrounding anatomic structures, particularly the trachea in a clockwise reference (Figure 1). Superior parathyroid adenomas are likely deeper in the neck in posterolateral positions compared with the trachea, and

A
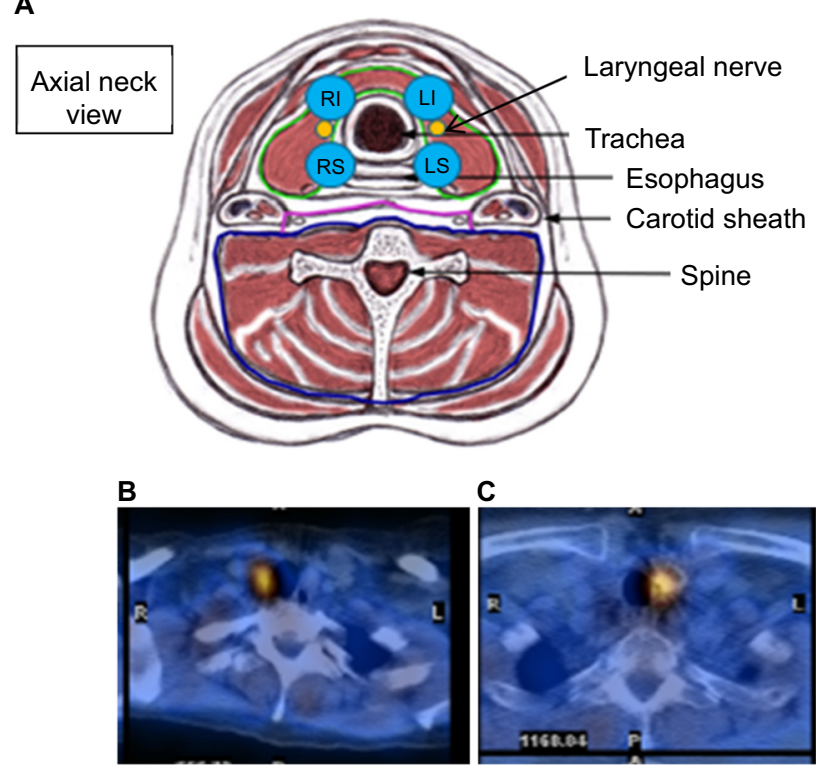

D

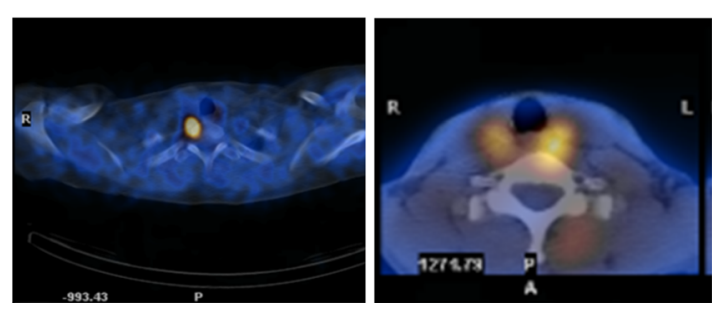

Figure I Schematic presentation of location of parathyroid adenomas in relation to the trachea (A) and examples of patients with perfect correlation with the intraoperative findings (B-E).

Abbreviations: RI, right inferior parathyroid; LI, left inferior parathyroid; RS, right superior parathyroid; LS, left superior parathyroid; L, left; R, right. inferior parathyroid adenomas are more superficial in the neck in anterolateral positions compared with the trachea.

\section{Parathyroid scan protocol}

Patients were injected intravenously with 20-25 mCi of Tc-99m sestamibi. Using low-energy high-resolution collimator and $10 \%$ window around the Technetium-99m photopeak (140 kev), anterior and left and right anterior oblique planar images were obtained at 20 minutes for 7 minutes and at 2-3 hours postinjection for 10 minutes each. These images were acquired into a $128 \times 128$ matrix with 1.6 zoom. SPECT/CT images were obtained at 45-60 minutes postinjection using the following parameters: $360^{\circ}$ arch, $3 \%$ step, 120 images, average time per stop is 27 seconds $\pm 1-3$ seconds as needed according to patient size and activity. The CT part is acquired simultaneously with the SPECT images using axial mode ( $5 \mathrm{~mm}$ interval), $140 \mathrm{kV}, 2.5 \mathrm{~mA}$, full rotation. The associated reconstruction parameters are $512 \times 512$ matrix, $1 \mathrm{~mm}$ pixel size, bone filter, and extended field of view.

\section{Statistical analysis}

Descriptive statistics was used to analyze the data collected using mean, median, and percentages. Correlation between image findings and postoperative pathological findings as the gold standard was also expressed as percentages of all the patients included in the study.

\section{Results}

The total study population consisted of 53 patients, 9 males and 44 females with an average age of $58.3 \pm 14.0$ years. The average preoperative serum calcium and PTH levels were $10.5 \pm 1.1 \mathrm{mg} / \mathrm{dL}$ and $165.4 \pm 79.8 \mathrm{pg} / \mathrm{mL}$, respectively. The mean and median interval between the scan and operative resection was 104 and 50 days, respectively. The correlation between SPS findings and surgical findings are summarized in Table 1. SPS interpretation correlated perfectly with surgical findings in $48 / 53$ patients $(90.6 \%)$. In three patients $(5.7 \%)$, SPS interpretation was on the correct side of the neck in correlation with surgical findings but not the exact

Table I Distribution of patients according to correlation between sestamibi parathyroid scan findings and surgical findings

\begin{tabular}{ll}
\hline $\begin{array}{l}\text { Parathyroid scan and surgical findings } \\
\text { correlation }\end{array}$ & Patients n (\%) \\
\hline $\begin{array}{l}\text { Perfect localization and surgical correlation } \\
\text { Same side of neck localization but not the exact }\end{array}$ & $48(90.6)$ \\
location & $3(5.6)$ \\
Nonlocalizing parathyroid scan & $2(3.8)$ \\
Total & $53(100)$ \\
\hline
\end{tabular}


location; that is, two patients SPS indicated a right superior parathyroid adenoma but intraoperatively they were inferior parathyroid adenoma and in the third patient, SPS indicated a left superior parathyroid adenoma, and intraoperatively it was left inferior adenoma. The correct location of the parathyroid adenoma was missed by SPS in two patients (3.7\%). Parathyroid adenoma was found intraoperatively on the opposite side in one of these two patients which was due to the coexistence of a multinodular thyroid gland interfering with the scan interpretation (Figure 2). Ten patients in our

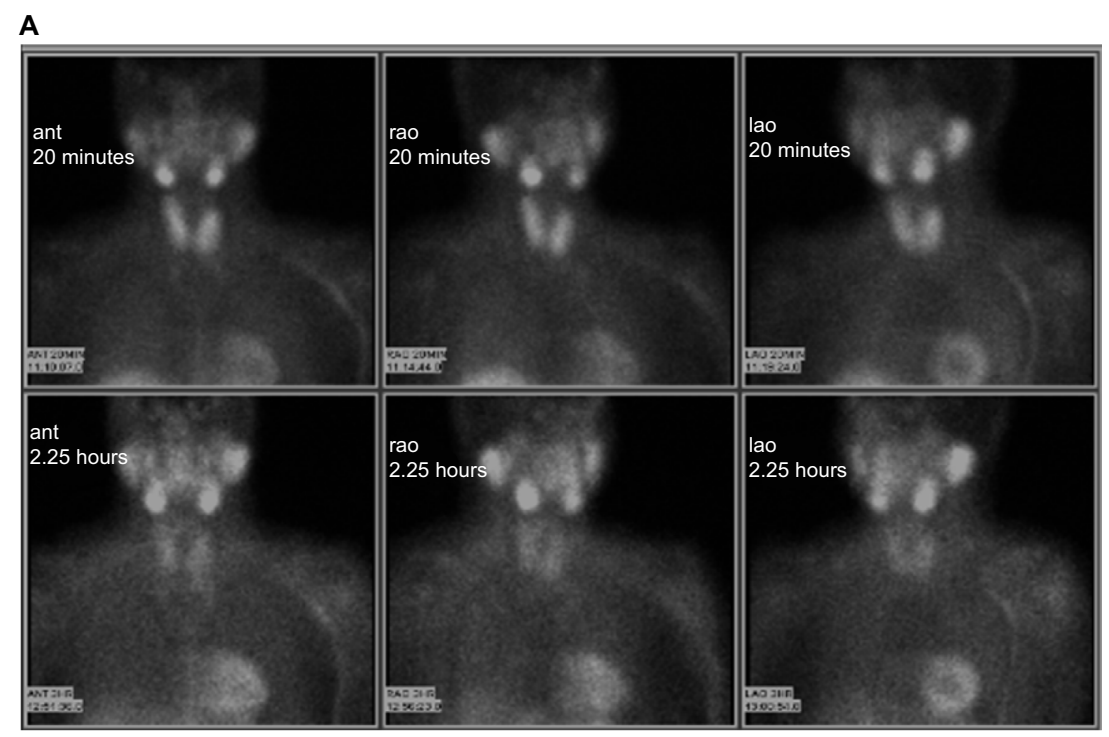

B

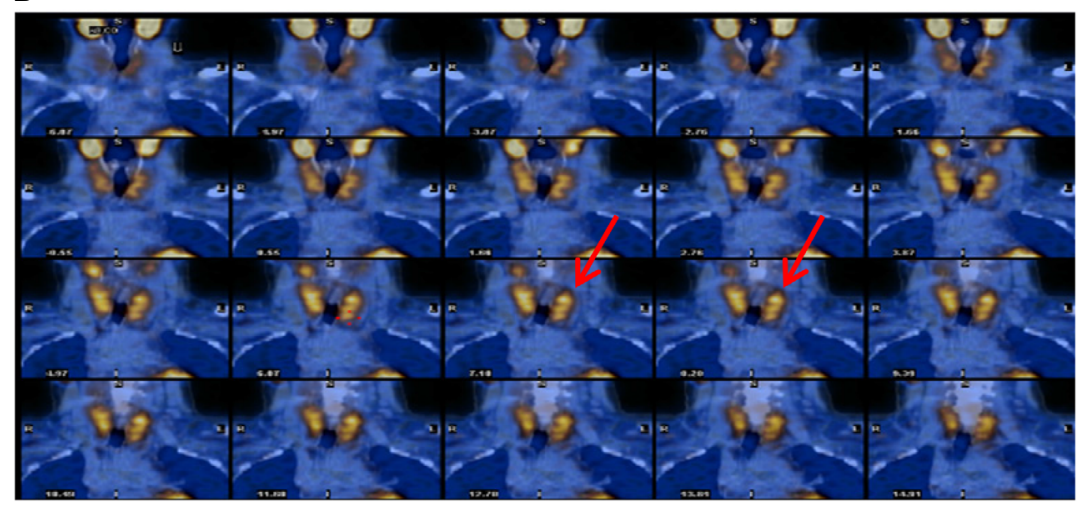

C

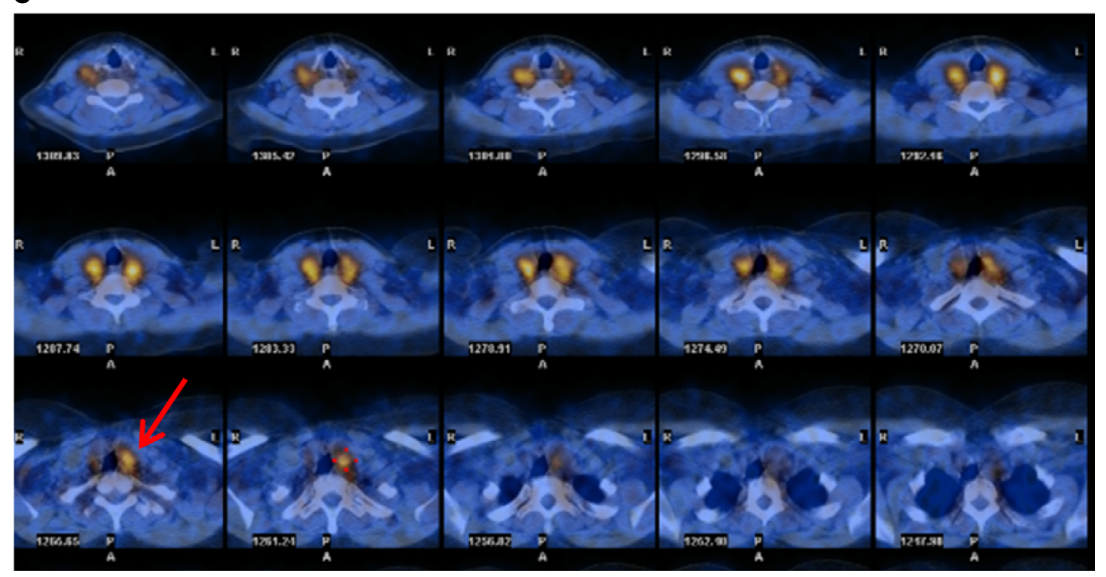

Figure 2 A nonlocalizing parathyroid scan with an enlarged multinodular thyroid gland. (A) A left superior parathyroid adenoma was suspected on the parathyroid scan (B, arrows), but a right inferior parathyroid adenoma was found intraoperatively (C, arrow).

Abbreviations: ant, anterior; rao, right anterior oblique; lao, left anterior oblique. 
study population were known to have coexisting multinodular thyroid gland, but this was the only patient with whom it interfered with the interpretation. The other patient with nonlocalizing SPS had an enlarged right submandibular gland which resulted in a false positive SPS, ultrasound, and 4D CT which resulted in removal of submandibular glandular tissue instead of the parathyroid adenoma (Figure 3). The average intraoperative PTH measurements decreased below $50 \%$ of the preoperative measurement with average postoperative level $39.7 \mathrm{pg} / \mathrm{mL}$, except in one patient where the adenoma was not found intraoperatively. Pathology confirmed the resection of the parathyroid adenoma in 52/53 patients $(98 \%)$.

The validation of accuracy of our interpretation method for localizing a parathyroid adenoma in relation to the trachea in 48 patients with perfect correlation is shown in Table 2.

\section{Discussion}

Single isotope dual-phase parathyroid scan had a major impact on the ease, accuracy, and utility of parathyroid scan for presurgical evaluation of patients with PHPT. ${ }^{17,18}$ The addition of SPECT and SPECT/CT technology represents the second most important advancements of parathyroid scintigraphy which has significantly facilitated minimally invasive parathyroidectomy. ${ }^{20-23,26}$ Multiple studies in the literature compare the performance of parathyroid scan to other anatomic imaging modalities..$^{10,24,25}$ Also in most institution, parathyroid scintigraphy is complemented with other anatomic imaging modalities; ultrasound, CT, and/or MRI for the localization of parathyroid adenomas. Our study aims to optimize the utility and interpretation of Tc- $99 \mathrm{~m}$ SPS with SPECT/CT for the diagnosis and localization of the abnormal parathyroid gland. Our results showed $90.6 \%$ accuracy of localization parathyroid adenoma site with a decrease in PTH level and pathologically confirmed parathyroidectomy. Although Quiros et al had lower accuracy results using SPS of $75 \%$ and $56 \%$ using ultrasound in a study included 71 patients, we both conclude that a positive SPS is the only preoperative required test in most patients with PHPT. ${ }^{27}$

Prior literature by Perrier et al described an alphabetical nomenclature of the different possible positions of parathyroid adenoma in the neck and in relation to the thyroid gland. This nomenclature assigns letters "A" through " $\mathrm{F}$ " for different surgical possible locations of the parathyroid adenoma. ${ }^{28}$ Perrier suggests that this alphabetical nomenclature system can facilitate communication between imagers and surgeons and can decrease ambiguity. Thus, it can

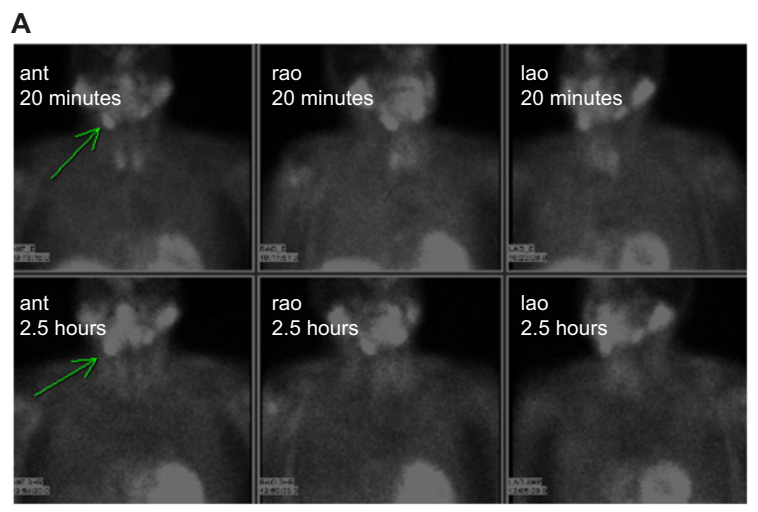

B

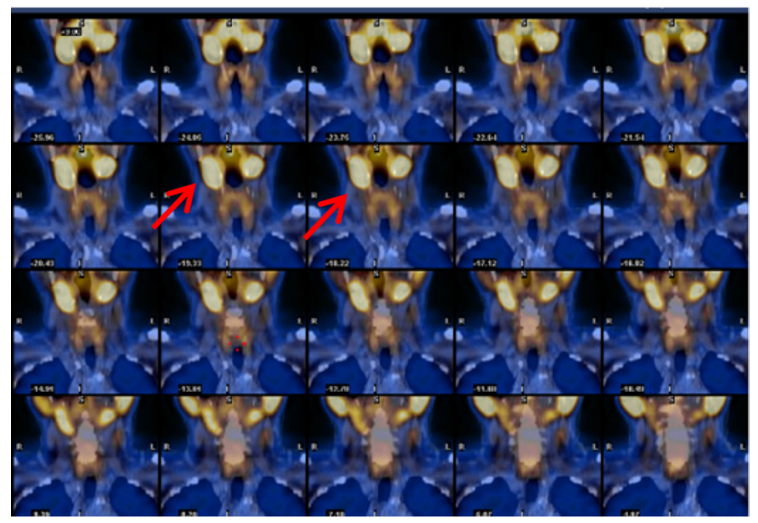

C

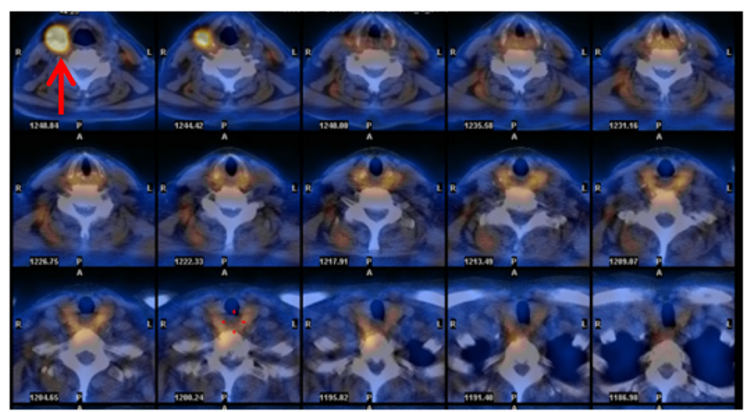

D

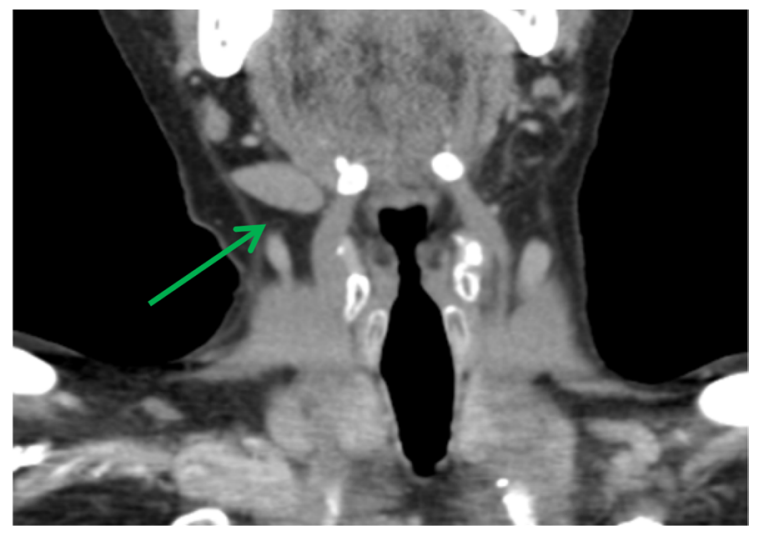

Figure $3 \mathrm{~A}$ patient with an enlarged right submandibular gland which was misleading as an ectopic right parathyroid adenoma on the SPS (A-C) and 4D CT scan (D). Arrows indicate an asymmetrically enlarged right submandibular gland resulting in a false positive SPS and CT scan.

Abbreviations: 4D, four dimensional; CT, computerized tomography; SPS, sestamibi parathyroid scan; ant, anterior; rao, right anterior oblique; lao, left anterior oblique. 
Table 2 Frequency of parathyroid gland involvement in clockwise locations around the trachea in patients with exact correlations between sestamibi parathyroid scan (SPS) and surgical findings

\begin{tabular}{lll}
\hline $\begin{array}{l}\text { Parathyroid gland } \\
\text { involved }\end{array}$ & $\begin{array}{l}\text { SPS location in relation } \\
\text { to trachea }\end{array}$ & Patient n (\%) \\
\hline $\begin{array}{l}\text { Left inferior } \\
\text { Left superior }\end{array}$ & Left anterolateral I - 2 o'clock & $16(30.2)$ \\
Right superior & Left posterolateral - 5 o'clock & $8(15.1)$ \\
Right inferior & Right posterolateral - 7o'clock & $10(18.9)$ \\
Total & Right anterolateral - 10 o'clock & $14(26.4)$ \\
\hline
\end{tabular}

easily guide the surgeon to the localization of the abnormal parathyroid intraoperatively. However, this nomenclature system can be used surgically, but it is difficult to apply to parathyroid scintigraphy because of the ballooning effects of radiotracer uptake in the abnormal parathyroid gland in addition to the limited resolution of gamma camera to structures $<1 \mathrm{~cm}$, which makes the nomenclature inapplicable in many instances when interpreting SPS and difficult to use to describe the exact location of a parathyroid adenoma. Particularly, it is difficult to differentiate type A from $\mathrm{B}$ adenomas, and $\mathrm{C}$ from $\mathrm{D}$ or $\mathrm{E}$ adenomas on SPS. In our study, we describe a more relevant and simple method of identifying and localizing the abnormal parathyroid adenoma in relation to the trachea in a clockwise fashion and in relation to the thyroid in a cranio-caudal level. Our interpretation method facilitates the reporting and communication between the imager and the surgeon, which translates into easier and faster localization of the abnormal gland intraoperatively with less invasive procedure and smaller operative scar. The abnormal parathyroid adenoma may also be localized with a clockwise numbering system around the trachea in a similar fashion to breast abnormalities when reported on ultrasound or mammogram. Thus, a right inferior parathyroid adenoma is usually located at the right anterolateral position relative to the trachea at 10 o'clock position, the left inferior parathyroid adenoma is located at the left anterolateral position at 2 o'clock relative to the trachea, the left superior is located at the left posterolateral position relative to the trachea at 5 o'clock, and the right superior is located in the right posterolateral position relative to the trachea at 7 o'clock (Figure 1). In addition, the level of the parathyroid adenoma level would be described in relation to the thyroid lobes in a cranio-caudal level. Our proposed interpretation methods identify the abnormal inferior versus superior parathyroid adenoma by its depth in relation to the trachea rather than by its position in relation to the thyroid, since many superior parathyroid adenomas tend to grow inferiorly behind the thyroid gland in the trachea-esophageal groove. ${ }^{29}$ Our results support the robustness and high accuracy of our method of interpretation and localization with $90.6 \%$ accuracy in identifying the exact location of the abnormal parathyroid gland correctly and $96.3 \%$ accuracy in identifying the laterality of the involved parathyroid gland. Our results also confirm superior results from a large meta-analysis study performed by Treglia et al which included pooled data from 23 studies for 1236 patient with Tc-99m MIBI SPECT/CT detection rate of $88 \%$ both on a per patient and per lesion analysis. ${ }^{30}$

A limitation of our study is the fact that it is a retrospective study, and not all patients who underwent parathyroid scans also underwent operative resection. Most likely, surgical management was not indicated in these patients. It is unlikely that it was due to nonlocalization or indeterminate parathyroid scan, since all patients without surgical management had a localizing parathyroid scan reports.

Although 10 patients in our study population were known to have multinodular thyroid gland, only one patient had a nonlocalizing SPS due to the interference with thyroid nodules. This is probably one of the strength of using SPECT/ CT as an integral part of SPS with better localization of focal abnormalities to the thyroid or outside the thyroid.

Our technique for localizing the abnormal parathyroid gland may be achieved in CT and MRI interpretation for parathyroid adenomas, although not tested in this study. However, the similarities between SPECT/CT, CT, and MRI in the tomographic nature of imaging would suggest the usefulness of our interpretation method if applied to the other tomographic imaging modalities.

\section{Conclusion}

Our study demonstrates high accuracy of Tc-99m sestamibi dual-phase scan with SPECT/CT in identifying the location of an abnormal parathyroid scan in PHPT using our systematic optimized interpretation method. Our optimized interpretation methods localize the parathyroid adenoma relative to the trachea and thyroid. A clockwise numerical position around the trachea can further simplify accurate localization of the parathyroid adenoma and facilitates communication between the imagers and the surgeons. Our results support the possible use of SPS as the sole diagnostic modality in most patients with primary PHPT with good surgical results.

\section{Acknowledgments}

The authors are thankful to Mrs Vicki Ephron for her support to this research project and Mrs Cheristena Bolos for her help in the production of this manuscript. 


\section{Disclosure}

The authors report no conflicts of interest in this work.

\section{References}

1. Al Zahrani A, Levine MA. Primary hyperparathyroidism. Lancet. 1997;349(9060):1233-1238.

2. Russell CF, Edis AJ. Surgery primary hyperparathyroidism: experience with 500 consecutive cases and evaluation of the role of surgery in the asymptomatic patient. Br J Surg. 1982;69(5):244-247.

3. Rubin MR, Bilezikian JP, McMahon DJ, et al. The natural history of primary hyperparathyroidism with or without parathyroidism surgery after 15 years. J Clin Endocrinol Metab. 2008; 93(9):3462-3470.

4. Greene AB, Butler RS, McIntyre S, et al. National trends in parathyroid surgery from 1998: a decade of change. J Am Coll Surg. 2009;209(3):332-343.

5. Udelsman R, Åkerström G, Biagini C, Duh QY, Miccoli P, Niederle B, Tonelli F. The surgical management of asymptomatic primary hyperparathyroidism: proceedings of the fourth international workshop. $J$ Clin Endocrinol Metab. 2014;99(10):3595-3606.

6. Henry JF, Sebag F, Cherenko M, Ippolito G, Taieb D, Vaillant J. Endoscopic parathyroidectomy: why and when? World J Surg. 2008;32(11):2509-2515.

7. Noda S, Onoda N, Kashiwagi S, et al. Strategy of operative treatment of hyperparathyroidism using US scan and (99m)Tc-MIBI SPECT/CT. Endocr J. 2014;61(3):225-230.

8. Ozkaya M, Elboga U, Sahin E, et al. Evaluation of conventional imaging techniques on preoperative localization in primary hyperparathyroidism. Bosn J Basic Med Sci. 2015;15(1):61-66.

9. Kwon JH, Kim EK, Lee HS, Moon HJ, Kwak JY. Neck ultrasonography as preoperative localization of primary hyperparathyroidism with an additional role of detecting thyroid malignancy. Eur J Radiol. 2013;82(1):e17-e21.

10. Lubitz CC, Hunter GJ, Hamberg LM, et al. Accuracy of 4-dimensional computed tomography in poorly localized patients with primary hyperparathyroidism. Surgery. 2010;148(6):1129-1137.

11. Michel L, Dupont M, Rosière A, Merlan V, Lacrosse M, Donckier JE. The rationale for performing MR imaging before surgery for primary hyperparathyroidism. Acta Chir Belg. 2013;113(2):112-122.

12. Prades JM, Gavid M, Timoshenko AT, Richard C, Martin C. Endoscopic surgery of the parathyroid glands: methods and principles. Eur Ann Otorhinolaryngol Head Neck Dis. 2013;130(3):157-160.

13. Gencoglu EA, Aras M, Moray G, Aktas A. The effectiveness of lowdose versus high-dose $99 \mathrm{mTc}$ MIBI protocols for radioguided surgery in patients with primary hyperparathyroidism. Nucl Med Commun. 2014;35(4):398-404.

14. Jayender J, Lee TC, Ruan DT. Real-time localization of parathyroid adenoma during parathyroidectomy. N Engl J Med. 2015;373(1):96-98

15. Harrell RM, Bimston DN. Optimization of minimally invasive radio-guided parathyroidectomy: the importance of neck ultrasonography and intraoperative parathyroid hormone assay. Endocr Pract. 2008;14(7):856-862.
16. Rajaei MH, Oltmann SC, Adkisson CD, Elfenbein DM, Chen H, Carty $\mathrm{SE}, \mathrm{McCoy} \mathrm{KL}$. Is intraoperative parathyroid hormone monitoring necessary with ipsilateral parathyroid gland visualization during anticipated unilateral exploration for primary hyperparathyroidism: a two-institution analysis of more than 2,000 patients. Surgery. 2014;156(4):760-766.

17. Geatti O, Shapiro B, Orsolon PG, Proto G, Guerra UP, Antonucci F, Gasparini D. Localization of parathyroid enlargement: experience with technetium-99m methoxyisobutylisonitrile and thallium-201 scintigraphy, ultrasonography and computed tomography. Eur J Nucl Med. 1994;21(1):17-22.

18. Coakley AJ, Kettle AG, Wells CP, O'Doherty MJ, Collins RE. 99Tcm sestamibi - a new agent for parathyroid imaging. Nucl Med Commun. 1989;10(11):791-794.

19. Gallowitsch HJ, Mikosch P, Kresnik E, Unterweger O, Lind P. Comparison between $99 \mathrm{mTc}$-tetrofosmin/pertechnetate subtraction scintigraphy and $99 \mathrm{mTc}$-tetrofosmin SPECT for preoperative localization of parathyroid adenoma in an endemic goiter area. Invest Radiol. 2000;35(8):453-459.

20. Wong KK, Fig LM, Gross MD, Dwamena BA. Parathyroid adenoma localization with $99 \mathrm{mTc}$-sestamibi SPECT/CT: a meta-analysis. Nucl Med Commun. 2015;36(4):363-375.

21. Eslamy HK, Ziessman HA. Parathyroid scintigraphy in patients with primary hyperparathyroidism: $99 \mathrm{mTc}$ sestamibi SPECT and SPECT/ CT. Radiographics. 2008;28(5):1461-1476.

22. Lavely WC, Goetze S, Friedman KP, et al. Comparison of SPECT/CT, SPECT, and planar imaging with single- and dual-phase $(99 \mathrm{~m}) \mathrm{Tc}-$ sestamibi parathyroid scintigraphy. J Nucl Med. 2007;48(7):1084-1089.

23. Neumann DR, Obuchowski NA, Difilippo FP. Preoperative 123I/99mTcsestamibi subtraction SPECT and SPECT/CT in primary hyperparathyroidism. J Nucl Med. 2008;49(12):2012-2017.

24. Ebner Y, Garti-Gross Y, Margulis A, Levy Y, Nabrisky D, Ophir D, Rotman-Pikielny P. Parathyroid surgery: correlation between preoperative localization studies and surgical outcomes. Clin Endocrinol (Oxf). 2015;83(5):733-738.

25. Heiba SI, Jiang M, Rivera J, Genden E, Inabnet W 3rd, Machac J, Kostakoglu L. Direct comparison of neck pinhole dual-tracer and dual-phase MIBI accuracies with and without SPECT/CT for parathyroid adenoma detection and localization. Clin Nucl Med. 2015;40(6):476-482.

26. Gayed IW, Kim EE, Broussard WF, et al. The value of $99 \mathrm{mTc}$-sestamibi SPECT/CT over conventional SPECT in the evaluation of parathyroid adenomas or hyperplasia. $J$ Nucl Med. 2005;46(2):248-252.

27. Quiros RM, Alioto J, Wilhelm SM, Ali A, Prinz RA. An algorithm to maximize use of minimally invasive parathyroidectomy. Arch Surg. 2004;139(5):501-506

28. Perrier ND, Edeiken B, Nunez R, et al. A novel nomenclature to classify parathyroid adenomas. World J Surg. 2009;33(3):412-416.

29. Phitayakorn R, McHenry CR. Incidence and location of ectopic abnormal parathyroid glands. Am J Surg. 2006;191(3):418-423.

30. Treglia G, Sadeghi R, Schalin-Jäntti C, Caldarella C, Ceriani L1, Giovanella L1, Eisele DW. Detection rate of (99m) Tc-MIBI single photon emission computed tomography (SPECT)/CT in preoperative planning for patients with primary hyperparathyroidism: a metaanalysis. Head Neck. 2016;38(Suppl 1):E2159-E2172.
Reports in Medical Imaging

\section{Publish your work in this journal}

Reports in Medical Imaging is an international, peer-reviewed, open access journal publishing original research, reports, reviews and commentaries on all areas of medical imaging. The manuscript management system is completely online and includes a very quick and fair peer-review system, which is all easy to use.

\section{Dovepress}

Visit http://www.dovepress.com/testimonials.php to read real quotes from published authors. 